\title{
Investigation of Fiber Waviness in a Thick Glass Composite Beam Using THz NDE
}

\author{
Robert F. Anastasi \\ U.S. Army Research Laboratory, Vehicle Technology Directorate, AMSRD-ARL-VT-MD, \\ Nondestructive Evaluation Sciences Branch, NASA Langley Research Center, \\ MS 231, Hampton, VA 23681
}

\begin{abstract}
Fiber waviness in laminated composite material is introduced during manufacture because of uneven curing, resin shrinkage, or ply buckling caused by bending the composite lay-up into its final shape prior to curing. The resulting waviness has a detrimental effect on mechanical properties, therefore this condition is important to detect and characterize. Ultrasonic characterization methods are difficult to interpret because elastic wave propagation is highly dependent on ply orientation and material stresses. By comparison, the pulsed terahertz response of the composite is shown to provide clear indications of the fiber waviness. Pulsed Terahertz NDE is an electromagnetic inspection method that operates in the frequency range between $300 \mathrm{GHz}$ and $3 \mathrm{THz}$. Its propagation is influenced by refractive index variations and interfaces. This work applies pulsed Terahertz NDE to the inspection of a thick composite beam with fiber waviness. The sample is a laminated glass composite material approximately $15 \mathrm{~mm}$ thick with a 90 -degree bend. Terahertz response from the planar section, away from the bend, is indicative of a homogeneous material with no major reflections from internal plies, while the multiple reflections at the bend area correspond to the fiber waviness. Results of these measurements are presented for the planar and bend areas.
\end{abstract}

Keywords: Terahertz, Nondestructive Evaluation, Laminated Composite, Waviness

\section{INTRODUCTION}

Out-of-plane fiber waviness in laminated composite material is characterized by undulations in a layer or group of layers within a multilayer laminate. These laminate undulations are a consequence of uneven curing, resin shrinkage, or ply buckling caused by bending the composite lay-up into its final shape prior to curing. This waviness causes degradation of strength and fatigue life in structural applications, particularly under compressive loads [1-3], therefore this condition is important to detect and characterize. Ultrasonic nondestructive methods have been utilized to measure fiber waviness. These measurements use piezoelectric transducers in contact with the sample to generate and detect ultrasonic waves [46]. When an ultrasonic wave is incident at an interface it is partially transmitted and reflected and its energy is distributed into longitudinal and transverse or shear waves modes. This process repeats at other lamina interfaces which at various angles relative to the propagation direction result in multiple wave modes and paths making analysis of ultrasonic transducer signals difficult. By comparison, electromagnetic waves transmit and reflect at interfaces like the ultrasonic waves, but electromagnetic wave energy is not split thus making interpretation simpler. One method of inspecting a material with electromagnetic waves is pulsed Terahertz (THz) nondestructive evaluation (NDE) which can be considered the electromagnetic analogy to pulse ultrasonic methods.

Pulsed THz NDE is a relatively new inspection technology $[7,8]$ that offers a non-contact and high-resolution inspection method. Its frequency regime is between $300 \mathrm{GHz}$ and $3 \mathrm{THz}$ and it has a free space wavelength range of 1000 to 100 $\mu \mathrm{m}$. This is a region of the electromagnetic spectrum between the microwave and infrared bands. This method has found application for aerospace material NDE [9,10] and in other areas such as astronomy [11], security application for chemical, biological, and weapons detection [12-16], and medical applications [17,18].

The pulsed THz system used in this work has been described in detail elsewhere [19] and was initially designed to inspect up to $30 \mathrm{~cm}$ of Sprayed-On Foam Insulation on the External Tank of the Space Shuttle. The system was integrated with a spatial scanner, data acquisition, and control system that translated the THz transceiver along two 
dimensions, and acquired and stored the temporal waveforms at incremental scan points. The system consists of an 800 $\mathrm{nm}$ femtosecond laser source with a pulse rate of $75 \mathrm{MHz}$, a THz control unit with a mechanical scanning optical delay line, a transceiver head with transmitter/receiver antenna, lens for focusing the THz beam, and a computer. Fiber optics connect the laser source to the transceiver and fully contain the laser light, which allows for a freely movable transmitter and receiver. The transceiver for this system, shown in figure 1, is a transmitter and receiver in a confocal arrangement. This confocal arrangement uses a $15 \mathrm{~cm}$ diameter, $285 \mathrm{~mm}$ focal length lens to concentrate the $\mathrm{THz}$ energy on the sample to a spot diameter of approximately $3 \mathrm{~mm}$. The digitized time-domain signal is composed of 2048 points and has a duration of $320 \mathrm{ps}$, which corresponds to a propagation path length in air of $96 \mathrm{~mm}$.

This paper presents a preliminary study of the capability of pulsed THz NDE for inspecting a thick composite beam with fiber waviness. The sample is a laminated glass composite material with a 90-degree bend. In this application, sample characteristics are determined in the $\mathrm{THz}$ frequency band and the sample is scanned and analyzed in a manner similar to ultrasonic imaging. $\mathrm{THz}$ response of the planar section away from the bend and at the bend area (with the fiber waviness) will be shown.

\section{COMPOSITE SAMPLE RESPONSE TO THz}

A picture of the composite sample is shown in figure 2a, it is made of S2-glass / polyphenylene sulfide thermoplastic tape and has a 92-ply lay-up of $\left[(0 /+45 / 90 /-45)_{11} /(+45 /-45)\right]_{s}$ and was originally manufactured for a curved beam strength study. The sample is approximately $15 \mathrm{~mm}$ thick with a 90-degree bend. The apex area is approximately $25 \mathrm{~mm}$ thick with wavy sub-lamina layers. The top radius is about $3.18 \mathrm{~cm}$ and the bottom radius is about $4.45 \mathrm{~cm}$. This sample along with a cube cut from the left leg of the sample, that doesn't exhibit fiber-waviness, were examined. The fiber waviness in the apex region, shown in figure $2 b$, has two main undulations that are almost symmetric about the apex with minor undulations on either side, thus there are four peaks and three valleys in the waviness section.

$\mathrm{THz}$ signals through different faces of the cube were obtained by positioning the sample under the THz transceiver. The cube was raised above the optical table to eliminate echo interference between the sample back and optical table surfaces. Figure 3 shows a picture of the cube along with its axis orientation. The sample $x$ - and $y$-axes were defined to be in the plane of the lamina while the $\mathrm{z}$-axis was perpendicular to the lamina.

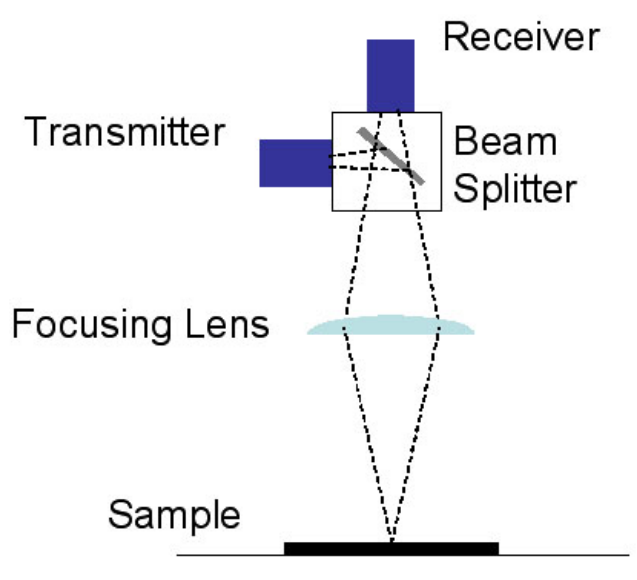

(a)

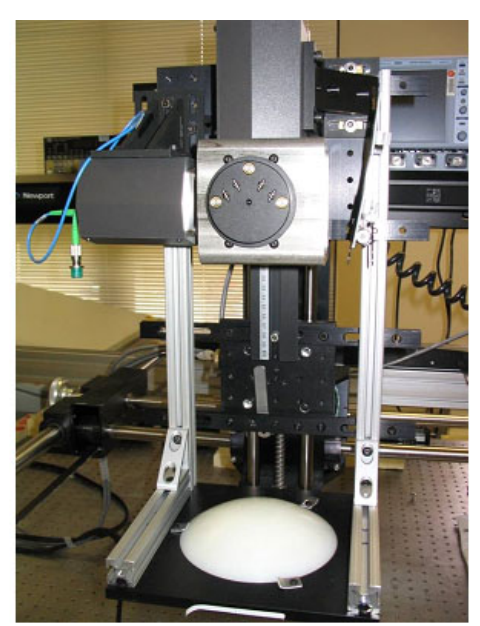

(b)

Figure 1. Transceiver confocal arrangement (a) schematic and (b) photograph. 


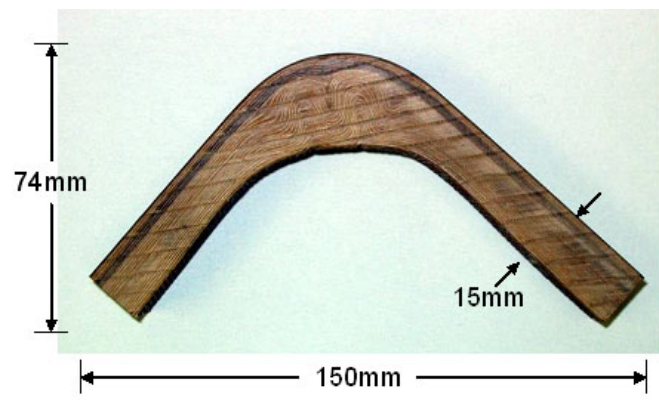

(a)

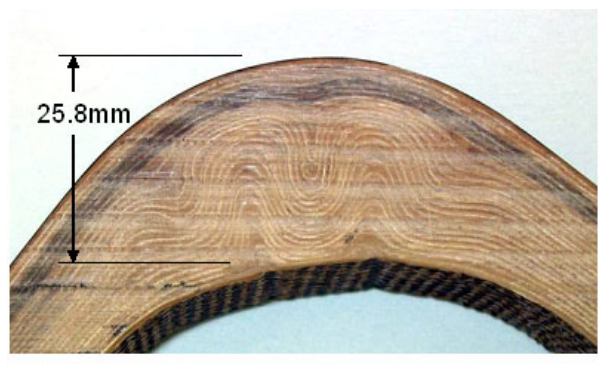

(b)

Figure 2. Composite sample with general dimensions (a) overall picture and (b) close-up of apex showing fiber-waviness

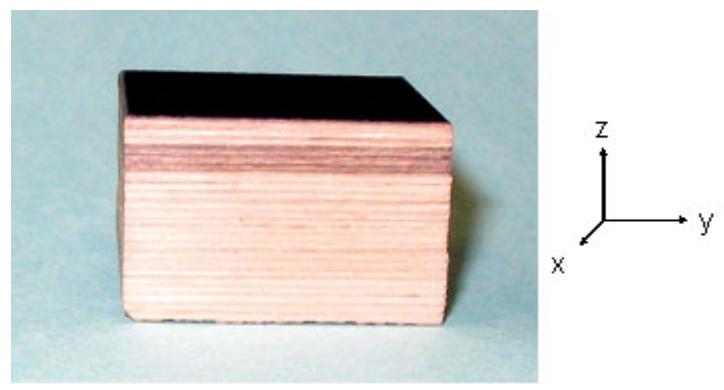

Figure 3. Picture of the composite cube and it axis orientation where the $\mathrm{x}$ - and $\mathrm{y}$-axes are in the plane of the lamina and the $\mathrm{z}$ axis is perpendicular to the lamina.

After inspecting the cube, the curved beam was examined in different orientations. The sample was positioned to inspect the left leg. In this position the direction of the THz beam propagation was parallel to the z-axis through the sample left leg. In a similar orientation the right leg was examined. To inspect the apex, the sample was positioned upright such that the $\mathrm{THz}$ propagation path was parallel to the z-axis through the apex. The last orientation was with the curved beam lying flat on the table surface; in this position the $\mathrm{THz}$ beam propagation path was parallel to the sample $\mathrm{x}$-axis.

For all the measurements, the THz transceiver height was adjusted to focus it at the sample top surface, the first incident surface. In some sample orientations, echoes from the sample top and back surfaces could not be seen on the same $\mathrm{THz}$ signal because of sample thickness. In these cases, two signals were recorded; one showing the front wall echo and one showing the back wall echo. These two signals were then combined into one composite signal that showed echoes from both the sample top and back surfaces. This was also done for imaging scans; one scan was conducted before and one after increasing the system time delay and then the two scans were combined into one composite scan.

\section{RESULTS}

THz signals through the cube are shown in figure 4. These signals show echoes from the sample top, back, and optical table surface (on which the scanner is mounted). To characterize the cube, $\mathrm{THz}$ signals and cube thickness measurements along each axis were used to calculate wave velocity, attenuation, and refractive index. The results, summarized in Table I, show the calculated values for each axis are all approximately equal. The average values are wave velocity of $1.47 \pm$ $0.01 \times 10^{8} \mathrm{~m} / \mathrm{s}$, attenuation of $1.01 \pm 0.01 \mathrm{~dB} / \mathrm{mm}$ including interface losses, and refractive index of $2.04 \pm 0.01$. Thus the cube's electromagnetic properties are approximately homogeneous with respect to the given $\mathrm{THz}$ frequency range. 
(a)

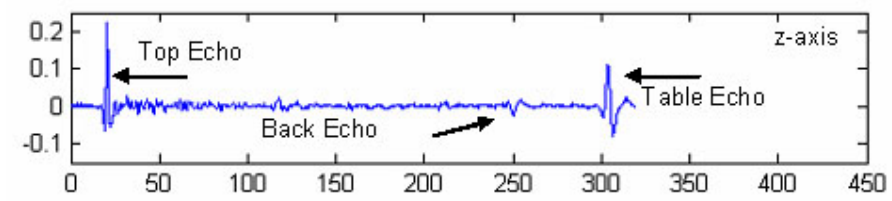

(b)

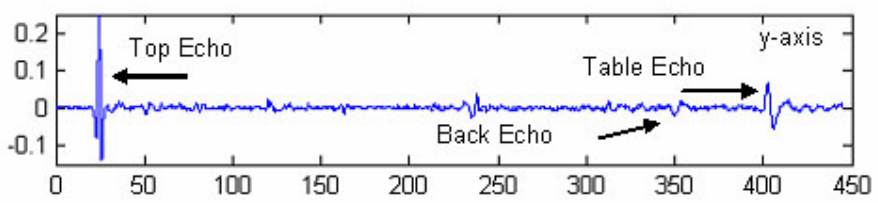

(c)

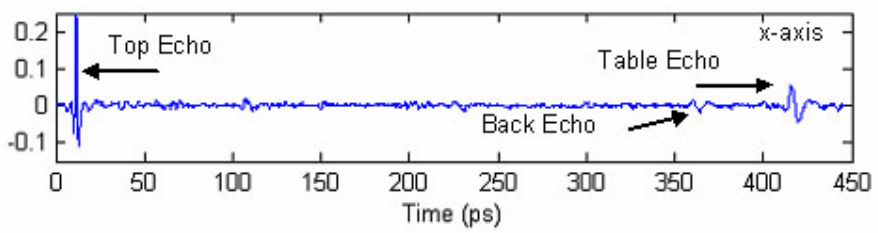

Figure 4. THz signals through the cube (a) z-axis, (b) y-axis, and (c) x-axis.

Table I. Summary of cube measurements

\begin{tabular}{|c|c|c|c|c|}
\hline Axis & $\begin{array}{c}\text { Thickness } \\
(\mathrm{mm})\end{array}$ & $\begin{array}{c}\text { Velocity } \\
\left(\mathrm{x} 10^{8} \mathrm{~m} / \mathrm{s}\right)\end{array}$ & $\begin{array}{c}\text { Attenuation Loss } \\
(\mathrm{dB} / \mathrm{mm})\end{array}$ & $\begin{array}{c}\text { Refractive } \\
\text { Index }\end{array}$ \\
\hline $\mathrm{X}$ & $23.99 \pm 0.01$ & $1.48 \pm 0.01$ & $1.02 \pm 0.01$ & $2.03 \pm 0.01$ \\
\hline $\mathrm{Y}$ & $26.23 \pm 0.01$ & $1.47 \pm 0.01$ & $0.99 \pm 0.01$ & $2.04 \pm 0.01$ \\
\hline $\mathrm{Z}$ & $17.01 \pm 0.02$ & $1.47 \pm 0.01$ & $1.03 \pm 0.01$ & $2.05 \pm 0.01$ \\
\hline
\end{tabular}

Scans of the curved sample, left and right leg, did not expose any internal defects. A picture of the sample orientation during inspection of the left leg and resulting C- and B-scan images are shown in figure 5. The C-scan in figure 5b is uniform in appearance and the B-scan in figure 5c exhibits some variation due, in part, to the sample layers and antenna noise.

THz images of the apex region shown in figure 6 are not uniform. The C-scan image in figure 6a shows four distinct areas of greater intensity which corresponds to the number of fiber waviness peaks. The B-scan image in figure 6b shows the apex echo and back surface echo. The back surface echo arrival time varies in a step-like manner and could be due to thickness variation or electromagnetic property variation. However, attributing the cause to thickness variation was ruled out because curvature and thickness vary gradually and not in a stepped fashion. Thus, the cause pointed to electromagnetic property changes in the apex region.

To inspect for the electromagnetic property variation in the apex region, the sample was laid flat on the table and scanned. The resulting $\mathrm{THz}$ C-scan amplitude image in figure 7 shows variations throughout the apex while the left and right legs are uniform. The lighter shades of gray correspond to greater back wall signal amplitude and the darker shades are less amplitude. Visual comparison of the THz image to the sample, suggests that the areas of greater back wall signal amplitude correspond to regions were the glass layers are further apart or where the fiber/matrix ratio is lower. Thus the glass fiber material reduces THz signal amplitude and areas with more matrix material are areas with greater THz signal amplitude. Matrix material samples or samples with various fiber/matrix ratios were not available to verify this observation. Refractive index of the left leg was found to be 2.06 while refractive index in a bright spot in the apex was 2.04 which is approximately a $2 \%$ difference. 


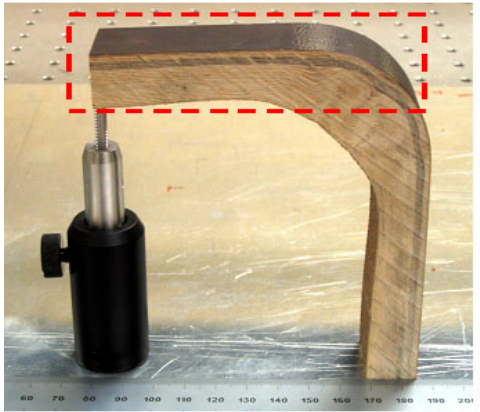

(a)

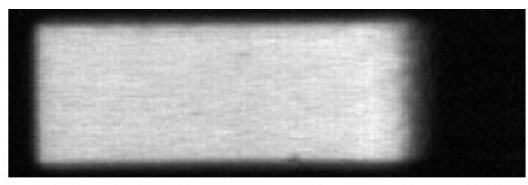

(b)

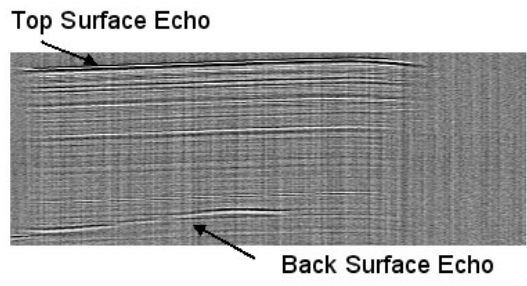

(c)

Figure 5. Sample left-leg scan (a) position of sample with outlined area indicating the section inspected, (b) THz Cscan amplitude image, and (c) THz B-scan image.

(a)
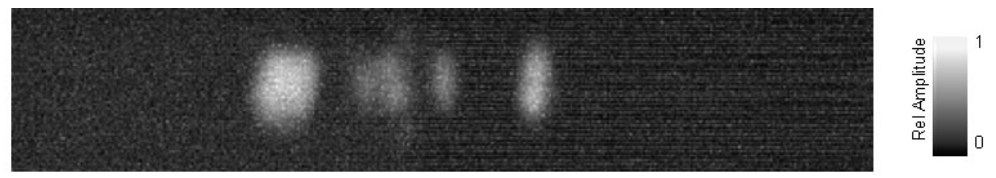

(b)
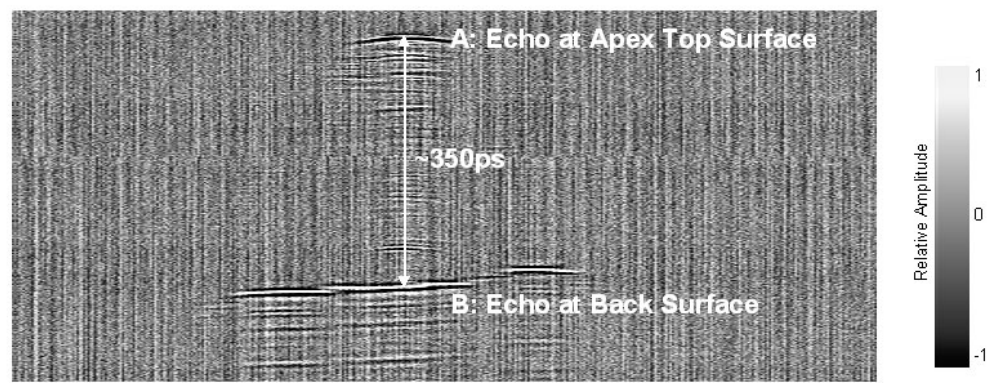

Figure 6. THz scans through apex with sample standing upright (a) THz C-scan, and (b) THz B-scan.

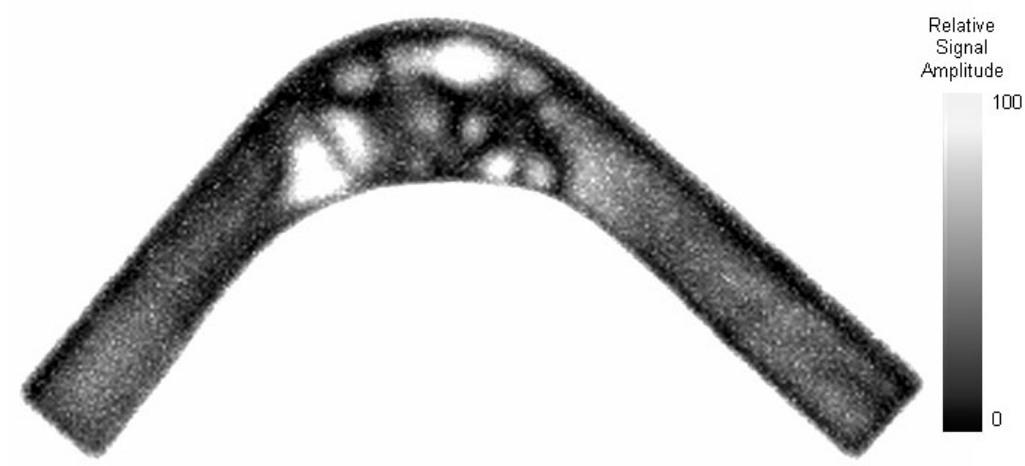

Figure 7. THz C-scan image with sample flat on table showing signal amplitude variations in the apex region 


\section{FINITE DIFFERENCE MODEL}

A finite difference time domain method was applied to simulate electromagnetic wave propagation [20] through the sample and highlight the influence of the sample's non-uniform electromagnetic properties. In this two-dimensional model Mur's absorbing boundary conditions [21] were used to prevent reflection from the domain edges, and the C-scan image in figure 7 was used as a refractive index map of the sample. The refractive index was considered to be realvalued with an absorption coefficient of zero and it was assumed the sample was non-magnetic and non-conductive. Thus the real refractive index was the only electromagnetic property of the sample considered. Excitation was a line source along the top edge of the domain with a half-cosine temporal profile and was easily implemented as opposed to the focused wave front of the THz transceiver. Excitation frequency was $0.5 \mathrm{THz}$ and corresponds to a wavelength $\lambda=$ $0.6 \mathrm{~mm}$ in free space. To reduce model complexity, the refractive index map of 256 gray levels was divided into five levels. The background was assigned a refractive index of 1.0 and four gray levels in the sample were assigned index values of 1.7, 1.8, 1.9, and 2.0. This corresponds to $\sim 20 \%$ difference in refractive index. To conserve computer memory and shorten computations duration the domain of the problem was reduced by considering only the apex area in the image and its overall dimensions were scaled down.

An example of a plane wave propagating forward through the uniform and non-uniform model is shown in figure 8. Figure 8a shows the uniform sample with a uniform wave front and figure 8b shows the non-uniform sample and nonuniform wave front resulting from material refractive index variations. As the wave continues to propagate it is reflected and transmitted at the sample back surface. Images of the wave after reflection from the back surface are shown in figures 9 and 10. Figure 9a shows the wave front near the top of the sample, figure 9b shows the wave starting to emerge from the sample, and figure 9c shows the wave completely emerged from the sample. In all cases the shape of the wavefront is smooth and uniform. Results for the non-uniform sample are shown in figure 10. Figure 10a shows the wave heading toward the sample top surface, figure 10b shows the wave starting to emerge, and figure 10c shows the wave completely emerged. In all cases the shape of the wave-front is no longer smooth and uniform. Wave front amplitude in the non-uniform sample is reduced due to multiple reflections within the material.

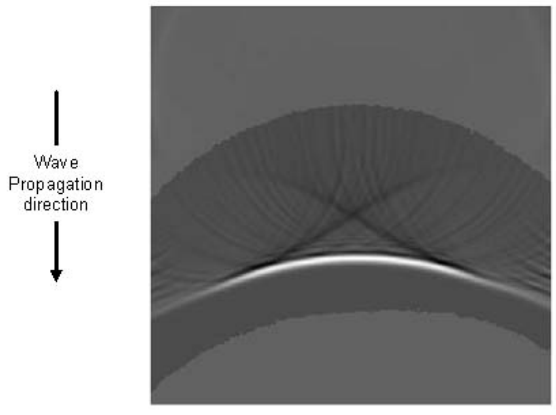

(a)

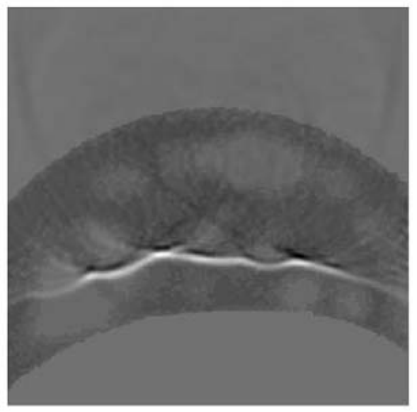

(b)

Figure 8. Result of the finite difference model forward propagation (a) uniform sample and (b) non-uniform sample.

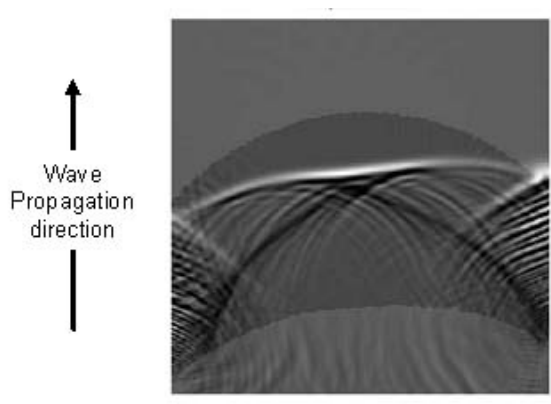

(a)

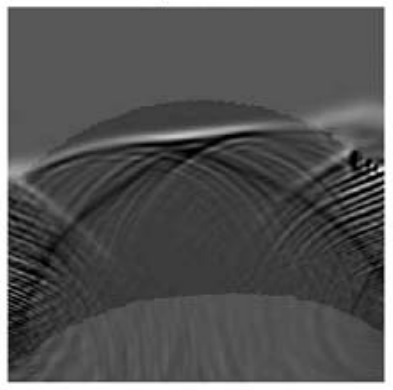

(b)

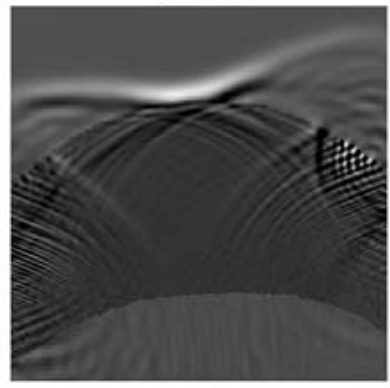

(c)

Figure 9. Result of the finite difference model for the uniform sample (a) wave near the top of the sample, (b) wave starting to emerge, and (c) wave emerged from sample. The wave-front is smooth or uniform. 


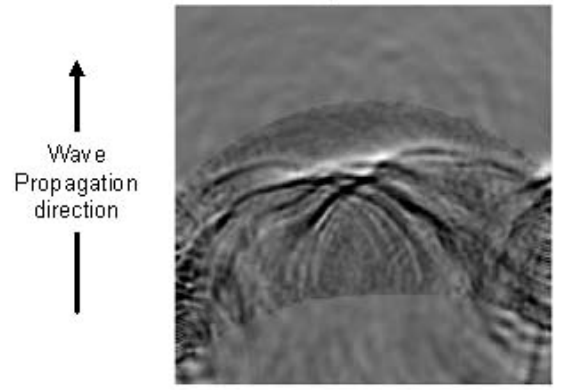

(a)

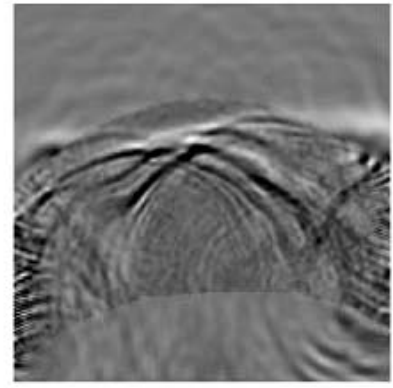

(b)

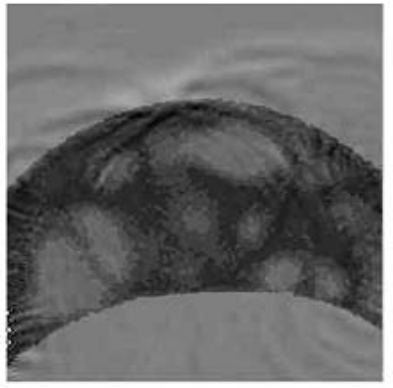

(c)

Figure 10. Result of the finite difference model for the non-uniform sample (a) wave near the top of the sample, (b) wave starting to emerge, and (c) wave emerged from sample. The wave-front is not smooth and not uniform.

\section{SUMMARY}

Electromagnetic properties of a curved composite sample with fiber waviness were measured in the $\mathrm{THz}$ regime and were found to be nearly identical in the plane of the plies and perpendicular to the plies. This showed that the sample was homogeneous with respect to electromagnetic wave propagation in the given $\mathrm{THz}$ frequency range. However, additional scans in the apex region illustrated that the sample was not homogeneous and that this non-homogeneity was a function of fiber/matrix ratio. A $2 \%$ difference was measured between the uniform leg areas and bright spots in the apex. To illustrate the effect of this non-homogeneity a finite difference wave propagation model was constructed with uniform and non-uniform refractive index cross-sections. A $20 \%$ difference in refractive index was used in the nonuniform model because smaller differences did not illustrate the effect due in part to model scaling and sensitivity. Results showed how the wave front in the uniform model was smooth while in the non-uniform model the wave front was not smooth and resulted from the non-uniform refractive index cross-section. The overall shape of the wave front in the model did not compare directly to the temporal location of the back surface echo in the B-scan image due in part to source excitation differences, a focused wave front in the measurement versus a line source in the model. However irregularities in both echo and wave front suggest refractive index relates to fiber/matrix ratio, fiber waviness, and shows that the one-sided THz NDE measurement can detect fiber waviness via fiber matrix ratio effects.

\section{REFERENCES}

1. Bogetti, T.A., J.W. Gillespie, Jr., and M.A. Lamontia, "Influence of Ply Waviness on Stiffness and Strength Reduction of Composite Laminates,” U.S. Army Research Laboratory, Technical Report ARL-TR-110, 1993

2. Murri, G.B., "Influence of Ply Waviness on Fatigue Life of Tapered Composite Flexbeam Laminates,” U.S. Army Research Laboratory, Technical Report ARL-TR-2110 and NASA Langley Research Center, TM-1999-209830, 1999

3. Wisnom, M.R. and J. W. Atkinson, "Fiber Waviness Generation and Measurement and Its Effects on Compressive Strength," Journal of Reinforced Plastics and Composites, Vol. 19, No. 02, 2000

4. Dayal, V., "Wave Propagation in a Composite with a Wavy Sublamina,” Journal of NDE, Vol 14, No. 1, 1995

5. Wooh, S. and I.M. Daniel, "Wave Propagation in Composite Materials with Fibre Waviness,” Ultrasonics, Vol. 33, No. 1, 1995

6. Kim, K., W. Zou, and W. Sachse, "Wave propagation in a wavy fiber-epoxy composite material: Theory and experiment," J. Acoust. Soc. Am. 103 (5), May 1998

7. Mittleman, D., et al., “T-Ray Tomography,” Optics Letters, Vol. 22, No. 12, June 1997

8. Dorney, T., et al., "Imaging with Terahertz Pulses,” International Symposium on Optical Science and Technology, July 2000

9. Anastasi, R.F., E.I. Madaras, J.P. Seebo, and W.P. Winfree, "Terahertz NDE for Aerospace Applications," published in "Ultrasonic and Advanced Methods for Nondestructive Testing and Material Characterization,” Edited by C.H. Chen, published by World Scientific Publishing, June 2007

10. Zimdars, D., J.S. White, G. Stuk, and G. Fichter, "Large Area Time Domain Terahertz (T-Ray) Imaging Non-Destructive Evaluation for Security and Aerospace," published in "Ultrasonic and Advanced Methods for Nondestructive Testing and Material Characterization,” Edited by C.H. Chen, published by World Scientific Publishing, June 2007

11. Woolard, D.L., E.R. Brown, M. Pepper, and M. Kemp, “Terahertz Frequency Sensing and Imaging: A Time of Reckoning Future Applications,” Proceedings of IEEE, Vol. 93, No. 10, pp 1722-1743, (2005) 
12. Fitch, M.J., C. Dodson, D.S. Ziomek, and R. Osiander, "Time-Domain Terahertz Spectroscopy of bio-agent Simulants," Chemical and Biological Standoff Detection II, edited by James O. Jensen, Jean-Marc Thériault, Proceedings of SPIE, Vol. 5584, pp 16-22, (2004)

13. Kawase, K., Y. Ogawa, and Y. Watanabe, "Non-Destructive Terahertz Imaging of Illicit Drugs Using Spectral Fingerprints,”Optics Express, Vol. 11, No. 20, pp 2549-2554, (2003)

14. Kemp, M.C., P.F. Taday, B.E. Cole, A.J. Fitzgerald, and W.R. Tribe, "Security Applications of Terahertz Technology," Terahertz for Military and Security Applications, R.J. Hwu, and D.L. Woolard editors, Proceedings of SPIE Vol. 5070, pp 44-52, (2003)

15. Anscombe, N., “No Place to Hide,” IEE Review, pp 26-30, (2005)

16. Zimdars, D., J.S. White, G. Stuk, A. Chemovsky, G. Fichter, and S. Williamson, "Large Area High Speed Time Domain THz Imager for Security and Non-Destructive Evaluation Imaging”THz Technology, Ultrafast Measurements, and Imaging, IEEE, pp 5-6, (2005)

17. Humphreys, K., J. P. Loughran, M. Gradziel, W. Lanigan, T. Ward, J.A. Murphy, C. O’Sullivan, "Medical applications of Terahertz Imaging: a Review of Current Technology and Potential Applications in Biomedical Engineering, ” Proceedings of the 26th Annual International Conference of the IEEE EMBS, pp 1302-1305, (2004)

18. Pickwell, E., B. E. Cole, A. J. Fitzgerald, M. Pepper, and V. P. Wallace, "In Vivo Study of Human Skin Using Pulsed Terahertz Radiation,” Phys. Med. Biol., Vol. 49, pp 1595-1607, (2004)

19. Zimdars, D., Valdmanis, J. A., White, J. S., Stuk, G., Williamson, S., Winfree, W.P, and Madaras, E. I., "Technology and Applications of Terahertz Imaging Non-Destructive Examination: Inspection of Space Shuttle Sprayed On Foam Insulation,” Review of Progress in Quantitative Nondestructive Evaluation, Golden, Colorado, 25-30 July (2004)

20. Yee, K. S., “Numerical Solution of Initial Boundary Value Problems Involving Maxwell's Equations in Isotropic Media,” IEEE Trans. Antennas Propogat, Vol. AP-14, pp. 302-307, May (1966)

21. Mur, G., “Total-Field Absorbing Boundary Conditions for the Time-Domain Electromagnetic Field Equations,” IEEE Trans. Electromagn Compat, Vol. 40, No. 2, pp. 100-102, May (1998) 